\title{
Article \\ Generalized Model Reference Scheduling and Control Co-Design with Guaranteed Performance
}

\author{
Shunli Zhao ${ }^{1,2, *}$, Cong Zhang ${ }^{2}$ and Lei Shao ${ }^{2, *}$ \\ 1 Tianjin Key Laboratory for Control Theory \& Applications in Complicated System, \\ Tianjin University of Technology, Tianjin 300384, China \\ 2 School of Electrical Engineering and Automation, Tianjin University of Technology, Tianjin 300384, China; \\ zhangcong06@163.com \\ * Correspondence: shlzhao@tjut.edu.cn (S.Z.); shaolei555@163.com (L.S.)
}

Citation: Zhao, S.; Zhang, C.; Shao,

L. Generalized Model Reference Scheduling and Control Co-Design with Guaranteed Performance.

Electronics 2021, 10, 2695. https:/ / doi.org/10.3390/electronics10212695

Academic Editor: Maysam Abbod

Received: 29 September 2021

Accepted: 1 November 2021

Published: 4 November 2021

Publisher's Note: MDPI stays neutral with regard to jurisdictional claims in published maps and institutional affiliations.

Copyright: (c) 2021 by the authors. Licensee MDPI, Basel, Switzerland. This article is an open access article distributed under the terms and conditions of the Creative Commons Attribution (CC BY) license (https:/ / creativecommons.org/licenses/by/ $4.0 /)$.

\begin{abstract}
In this paper, a generalized model-reference scheduling (GMRS) scheme is proposed for a networked control system (NCS) with guaranteed performance control and medium access constraint (MAC). The GMRS presented reduces conservatism to some extent, and its performance is improved by adjusting the weighted gain. In addition, two cases of uncertainty existing in the system matrix and control matrix are considered. For the first case, a co-design of guaranteed performance control and the GMRS is studied for the NCS with and without zero-order hold mechanism. For the second case, the uncertainty induced from time delay is considered in the guaranteed performance control and the GMRS co-design. Finally, illustrative examples are given to demonstrate the effectiveness of the proposed co-design schemes.
\end{abstract}

Keywords: generalized model-reference scheduling; guaranteed performance control; system uncertainty; medium access constraint

\section{Introduction}

Different from the traditional control systems, where sensors, controllers, actuators, and plants are integrated, a NCS is a type of distributed control system where the elements are spatially decentralized through a network. This type of system enables wiring reduction and has easy maintenance. More and more attention has been drawn along with the improvement of new technologies such as computer science, control theory, and communication technology [1-3]. However, many challenges are rising with the introduction of the network, such as the issues of time delay, data packet dropout, data packet disorder, and so on [4-6].

As is known, bandwidth is a critical resource for a wired or wireless network. A great deal of information from sensors, actuators, and controllers is transmitted through a bandwidth-limited network in the NCS. How to effectively save the limited bandwidth is an open question that is worth thoroughly pondering. Until now, several useful bandwidthsaving strategies have been proposed. First of all, quantization is thought to be a passive bandwidth-saving method, as much redundant data are discarded in the signal transformation from analog to digital [7-10]. In [7], a quantized event-driven control approach is proposed to reduce the amounts of data for each transmission. A class of discrete-time Takagi-Sugeno (T-S) fuzzy NCS with time-varying delay and quantization effects is studied in [8]; the states are quantized before transmission via a logarithmic quantizer. The quantization effects are processed by using the generalized sector-bound approach. A new dynamic quantization strategy is presented to prevent saturation of the quantizer with external disturbances in [9]. In [10], an optimal tracking performance of multiple input multiple output (MIMO) NCS with quantization and bandwidth constraint is proposed to realize the optimal tracking performance and the bandwidth-saving as well. It is worth noting that quantization can improve the bandwidth utility to some extent. However, 
it also brings a new problem of missing data as the information between two adjacent quantization levels is dropped. This is obviously harmful to the performance of the NCS. Another bandwidth-saving approach, called an event-triggering mechanism (ETM), was proposed recently, and has become a hot topic. In an ETM, the information will be transmitted over the network when meeting some event-triggered conditions [11-13]. A dynamic decentralized periodic event-triggered control for wireless cyber-physical systems whose sensors are distributed and bandwidths are limited is studied in [11], and it can save more than $40 \%$ of events. A designed event-triggered control approach is proposed in [12]. A minimal spanning in-tree algorithm to minimize the triggering event set is suggested to realize the purpose of the bandwidth-saving. In [13], two event-triggering mechanisms are introduced to limit the number of packets sent over the network.

Besides the above two methods, an active scheduling scheme is another effective bandwidth-saving approach. It is initially introduced in solving MAC, where not all the data will be transmitted over the network due to the limited network access. The scheduling scheme determines which of the nodes can access the network. In this context, the scheduling scheme can be applied to save bandwidth by actively reducing the number of the nodes accessing the network, even though the access of the network is not the main concern. It is obvious that reducing the number of the nodes can result in a reduction in information transmitting over a network.

The scheduling scheme used in NCS was firstly introduced by CPU task scheduling in earlier years [14]. Two improved scheduling schemes, rate monotonic (RM) [15] and earliest deadline first (EDF) [16], are proposed to solve the non-preemptive NCS tasks. During the period, it is worth noting that the control algorithm and scheduling scheme are separately designed in NCS analysis and synthesis. This may be harmful to the improvement of the NCS performance. In order to solve this issue, a communication sequence-based scheduling scheme is proposed to provide an idea to fix the problem of the separate design [17-19]. The introduction of the communication sequence makes the control and scheduling co-design possible based on the one-to-one correspondence between the scheduling sequence and the states or control signals. A scheduling approach combing priority-based scheduling and the communication sequence, maximum error first and try once discard (MEF-TOD), is proposed in [20]. It has been widely used in several fields since it was put forward, such as in wireless multi-hop networks [21], a quantization control system [22], stochastic networked control [23], an automotive electronic valve system [24], a state-saturated system [25], and the references therein. However, it is worth noting that the MEF-TOD scheduling uses the errors of the states between adjacent periods. This may introduce conservatism, as more performance is thoughtless.

In order to reduce the conservatism, an initial version of model reference scheduling (MRS), called model-dependent scheduling (MDS), is proposed in [26], where the ideal dynamic is introduced, and the absolute value of the error between the real system and the ideal dynamic is used. Although this work is promising, it also has conservatism. Besides, only the uncertainty of the control matrix is considered in [26], while the uncertainty of the system matrix is not involved. Motivated by this, we propose a GMRS in this paper. The conservatism of the MDS is reduced by adding an adjustable weighted gain before the errors. In this context, the MDS becomes a special case of the GMRS. Therefore, the flexibility of the MDS is improved. In addition, not only the uncertainty of the control matrix but also the uncertainty of the system matrix induced by modeling errors is considered in the paper. Two cases of the processing of the sensor information, zero-order hold and non-zero-order hold, are analyzed when the state cannot gain the access to the network.

Additional steps toward more generalized MRS and performance consideration are certainly required. In order to realize the guaranteed performance, a co-design of the GMRS and the guaranteed performance control is proposed in this paper. The NCS with MAC and the uncertainty in system matrix and control matrix can be stabilized with a performance bound using the guaranteed performance control. The main contributions of this paper are summarized as follows: 
(1) Extending the modeling approach proposed in [26]; the uncertainty in the system matrix and the uncertainty in the control matrix are considered. Two representative cases of the processing of the sensor information, zero-order hold and non-zero-order hold, are analyzed when the state cannot gain the access to the network.

(2) Generalizing the MDS in [26]; the conservatism of the MDS is obviously decreased.

The organization of the remainder of the paper is as follows. The uncertainty in the system matrix and the uncertainty in the control matrix are described in Section 2. The principle of the GMRS is explained in Section 3. In Section 4, the guaranteed performance control and scheduling co-design procedures with the system uncertainties are detailed. Examples are presented to demonstrate the effectiveness of the proposed method in Section 5. Section 6 concludes the paper.

\section{Systems Description and Preliminaries}

MAC is an interesting study in NCS. States in the NCS with MAC are not totally transmitted to the controller side because of the scarce network bandwidth resources. The NCS with MAC is depicted in Figure 1. The states $x_{1} \cdots x_{n}$ are not delivered through the network simultaneously. Which of the states can access the network is dependent on the scheduling scheme. $\boldsymbol{\delta}(k)$ in Figure 1 means a scheduling vector used by the concrete scheduling scheme.

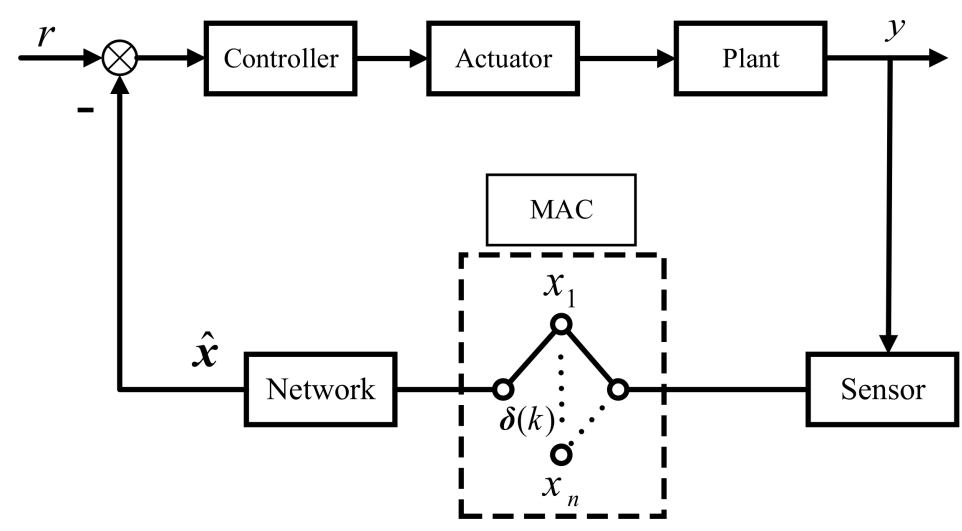

Figure 1. NCS with MAC.

Uncertainties in NCS involve two types from the prospect of system structures, i.e., the uncertainty in the system matrix and the uncertainty in the control matrix. The detailed analysis and design are studied as follows.

\subsection{System with Uncertainty in the System Matrix}

Uncertainty in the system matrix in NCS comes from the modeling uncertainty, such as errors of the modeling, loss of the complete identification for the working mechanism, and aging of the electronic components, and so on. There are two types of compensation mechanisms when not all the states can access the network for the NCS with the uncertainty in the system matrix in this paper. One is the system without a zero-order hold approach, and the other is the one with a zero-order hold mechanism.

\subsubsection{System without Zero-Order Hold}

NCS with the uncertainty in the system matrix can be described as:

$$
\left\{\begin{array}{l}
x(k+1)=(\boldsymbol{A}+\Delta \boldsymbol{A}) \boldsymbol{x}(k)+\boldsymbol{B} \boldsymbol{u}(k) \\
\boldsymbol{y}(k)=\boldsymbol{C} \boldsymbol{x}(k)
\end{array}\right.
$$

where $\boldsymbol{x}(k) \in \mathbf{R}^{n}, \boldsymbol{y}(k) \in \mathbf{R}^{r}$, and $\boldsymbol{u}(k) \in \mathbf{R}^{m}$ denote the state vector, system output, and control signal, respectively. $A, B$, and $C$ are constant matrices with appropriate dimensions. 
$\Delta \boldsymbol{A}=\boldsymbol{D F E}$, where $\boldsymbol{D}$ and $\boldsymbol{E}$ are constant matrices and $\boldsymbol{F}^{\mathrm{T}} \boldsymbol{F} \leq \boldsymbol{I}, \boldsymbol{I}$ is an identity matrix. The NCS without the zero-order hold is depicted in Figure 2.

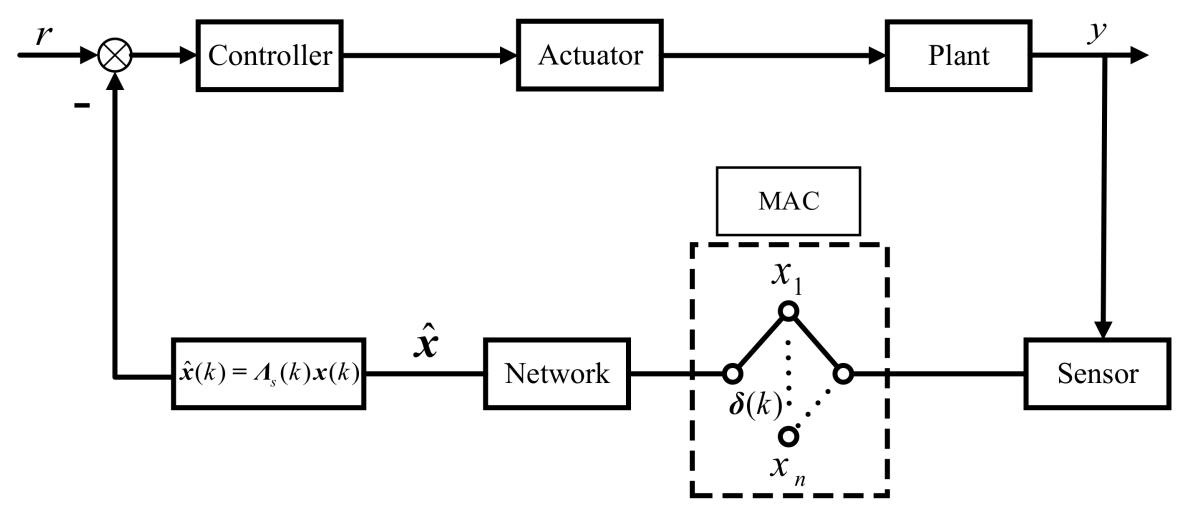

Figure 2. NCS with the MAC but without the zero-order hold.

As shown in Figure 2, MAC exists in the feedback loop of the NCS; the states that cannot gain the access to the network will be set to zero because of the NCS without the zero-order hold. The states transmitted from the sensor will not be totally received by the controller because of the MAC. Only parts of the states can be received by the controller, while the others are discarded, and their values are reset to zero in the controller side. In this context, the states received by the controller can be written as:

$$
\hat{x}(k)=\Lambda_{s}(k) x(k)
$$

where $\boldsymbol{\Lambda}_{s}(k)$ denotes the scheduling matrices composed of some scheduling vectors. Subscript $s$ means the different scheduling matrix.

In this context, the state feedback controller can be described as:

$$
\boldsymbol{u}(k)=\boldsymbol{K}_{s} \hat{\boldsymbol{x}}(k)=\boldsymbol{K}_{s} \boldsymbol{\Lambda}_{s} \boldsymbol{x}(k)
$$

Then, the system (1) can be written as the following equation:

$$
\boldsymbol{x}(k+1)=(\boldsymbol{A}+\Delta \boldsymbol{A}) \boldsymbol{x}(k)+\boldsymbol{B} \boldsymbol{K}_{s} \boldsymbol{\Lambda}_{s} \boldsymbol{x}(k)=\boldsymbol{\Phi}_{s} \boldsymbol{x}(k)
$$

where $\boldsymbol{\Phi}_{s}=A+\Delta A+B K_{s} \Lambda_{s}$.

\subsubsection{System with Zero-Order Hold}

Similar to Figure 2, the states transmitted from the sensor will not be totally received by the controller because of the MAC. Only parts of the states can be received by the controller, while the others are discarded but their values are reset to the last ones in the controller side. NCS with the zero-order hold can be depicted as in Figure 3.

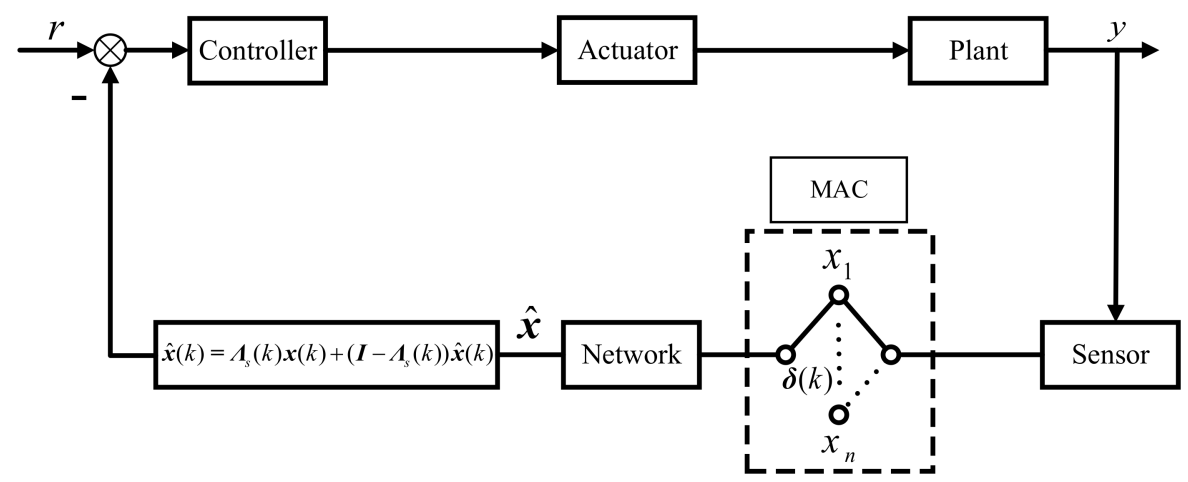

Figure 3. NCS with the MAC and the zero-order hold. 
From Figure 3, the MAC exists in the feedback loop of the NCS. Additionally, the states that cannot gain access to the network will be set to the last ones because of the zero-order hold mechanism. In this context, the states received by the controller can be written as:

$$
\hat{\boldsymbol{x}}(k)=\boldsymbol{\Lambda}_{s}(k) \boldsymbol{x}(k)+\left(\boldsymbol{I}-\boldsymbol{\Lambda}_{s}(k)\right) \hat{\boldsymbol{x}}(k-1)
$$

The state feedback controller can be represented as:

$$
\boldsymbol{u}(k)=\boldsymbol{K}_{s} \hat{\boldsymbol{x}}(k)=\boldsymbol{K}_{s} \boldsymbol{\Lambda}_{s} \boldsymbol{x}(k)+\boldsymbol{K}_{s}\left(\boldsymbol{I}-\boldsymbol{\Lambda}_{s}(k)\right) \hat{\boldsymbol{x}}(k-1)
$$

We let $z(k)=\left[\begin{array}{ll}x^{\mathrm{T}}(k) & \hat{x}^{\mathrm{T}}(k-1)\end{array}\right]^{\mathrm{T}}$; the system (1) can thus be rewritten as the following equation.:

$$
z(k+1)=\boldsymbol{\Theta}_{s} z(k)
$$

where $\boldsymbol{\Theta}_{s}=\left[\begin{array}{cc}\boldsymbol{A}+\Delta \boldsymbol{A}+\boldsymbol{B} \boldsymbol{K}_{s} \boldsymbol{\Lambda}_{s} & \boldsymbol{B} \boldsymbol{K}_{s}\left(\boldsymbol{I}-\boldsymbol{\Lambda}_{s}\right) \\ \boldsymbol{\Lambda}_{s} & \boldsymbol{I}-\boldsymbol{\Lambda}_{s}\end{array}\right]=\overline{\boldsymbol{A}}_{s}+\overline{\boldsymbol{D}} \boldsymbol{F} \overline{\boldsymbol{E}}+\overline{\boldsymbol{B}} \boldsymbol{K}_{s} \Delta_{s}$, and where $\bar{A}_{s}=\left[\begin{array}{cc}A & 0 \\ \boldsymbol{\Lambda}_{s} & \boldsymbol{I}-\boldsymbol{\Lambda}_{s}\end{array}\right], \overline{\mathbf{D}}=\left[\begin{array}{c}\boldsymbol{D} \\ 0\end{array}\right], \overline{\boldsymbol{E}}=\left[\begin{array}{ll}\boldsymbol{E} & 0\end{array}\right], \Delta_{s}=\left[\begin{array}{cc}\boldsymbol{\Lambda}_{s} & \boldsymbol{I}-\boldsymbol{\Lambda}_{s}\end{array}\right]$.

\subsection{System with Uncertainty in the Control Matrix}

Considering a continuous NCS, it is imposed by random time delay in the information transmission. Uncertainties are introduced because of the random character of the time delay. The continuous NCS can be given as:

$$
\left\{\begin{array}{l}
\dot{x}(t)=A x(t)+B \boldsymbol{u}(t) \\
\boldsymbol{y}(t)=C x(t)
\end{array}\right.
$$

Suppose that the time delay $\tau_{k}<T$, and $T$ is the sampling period of the NCS. Discretizing the system (8) with the time delay $\tau_{k}$ and the sampling period $T$, we can obtain the following system equation:

$$
\boldsymbol{x}(k+1)=\boldsymbol{G} \boldsymbol{x}(k)+\boldsymbol{H}_{0}\left(\tau_{k}\right) \boldsymbol{u}(k)+\boldsymbol{H}_{1}\left(\tau_{k}\right) \boldsymbol{u}(k-1)
$$

where $\boldsymbol{G}=\exp (\boldsymbol{A} T), \boldsymbol{H}_{0}\left(\tau_{k}\right)=\int_{0}^{T-\tau_{k}} e^{\boldsymbol{A} t} d t \cdot \boldsymbol{B}=\int_{0}^{T / 2} e^{\boldsymbol{A} t} d t \cdot \boldsymbol{B}+e^{\boldsymbol{A} T / 2} \int_{0}^{-\tau_{k}{ }^{\prime}} e^{\boldsymbol{A} t} d t \cdot \boldsymbol{B}$, and $\boldsymbol{H}_{1}\left(\tau_{k}\right)=\int_{T-\tau_{k}}^{T} e^{A t} d t \cdot \boldsymbol{B}=\int_{T / 2}^{T} e^{\boldsymbol{A} t} d t \cdot \boldsymbol{B}-e^{\boldsymbol{A} T / 2} \int_{0}^{-\tau_{k}{ }^{\prime}} e^{\boldsymbol{A} t} d t \cdot \boldsymbol{B}$.

We let $\delta=\max _{\tau^{\prime} \in[-T / 2, T / 2]}\left\|\int_{0}^{-\tau^{\prime}{ }^{\prime}} e^{A t} d t\right\|_{2}=\left\|\int_{0}^{\frac{T}{2}} e^{A t} d t\right\|_{2}, \overline{\boldsymbol{F}}\left(\tau_{k}{ }^{\prime}\right)=\int_{0}^{-\tau_{k}{ }^{\prime}} e^{A t} d t$, and $\boldsymbol{F}\left(\tau_{k}{ }^{\prime}\right)=\mathcal{\delta}^{-1} \overline{\boldsymbol{F}}\left(\tau_{k}{ }^{\prime}\right)$; then, we have $\boldsymbol{F}^{T}\left(\tau_{k}{ }^{\prime}\right) \boldsymbol{F}\left(\tau_{k}{ }^{\prime}\right)=\mathcal{\delta}^{-2} \overline{\boldsymbol{F}}^{T}\left(\tau_{k}{ }^{\prime}\right) \overline{\boldsymbol{F}}\left(\tau_{k}{ }^{\prime}\right) \leq \boldsymbol{I}$.

In this context, $\boldsymbol{H}_{0}\left(\tau_{k}\right)$ and $\boldsymbol{H}_{1}\left(\tau_{k}\right)$ can be written as $\boldsymbol{H}_{0}\left(\tau_{k}\right)=\Gamma_{0}+\boldsymbol{D F}\left(\tau_{k}^{\prime}\right) \widetilde{\boldsymbol{E}}$ and $\underset{\widetilde{\boldsymbol{E}}}{\boldsymbol{H}_{1}}\left(\tau_{k}\right)=\Gamma_{1}-\boldsymbol{D F}\left(\tau_{k}{ }^{\prime}\right) \widetilde{\boldsymbol{E}}$, where $\Gamma_{0}=\int_{0}^{T / 2} e^{\boldsymbol{A} t} d t \cdot \boldsymbol{B}, \Gamma_{1}=\int_{T / 2}^{T} e^{\boldsymbol{A t}} d t \cdot \boldsymbol{B}, \boldsymbol{D}=\delta e^{\boldsymbol{A}(T / 2)}$, $\widetilde{E}=\boldsymbol{B}$.

We let $\boldsymbol{v}(k)=\left[x^{\mathrm{T}}(k) \hat{\boldsymbol{x}}^{\mathrm{T}}(k-1) \boldsymbol{u}^{\mathrm{T}}(k-1)\right]^{\mathrm{T}}$, and use the same state feedback controller $\boldsymbol{u}(k)=\boldsymbol{K}_{s} \hat{\boldsymbol{x}}(k)$ as before, then the system (9) can be closed by:

$$
\boldsymbol{v}(k+1)=\mathbf{\Omega}_{s} \boldsymbol{v}(k)
$$

where $\begin{array}{rcc}\boldsymbol{\Omega}_{s} & =\left[\begin{array}{ccc}\boldsymbol{G}+\left(\boldsymbol{H}_{0}+\boldsymbol{D} \boldsymbol{F}\left(\tau_{k}^{\prime}\right) \widetilde{\boldsymbol{E}}\right) \boldsymbol{K}_{s} \boldsymbol{\Lambda}_{s} & \left(\boldsymbol{H}_{0}+\boldsymbol{D} \boldsymbol{F}\left(\tau_{k}^{\prime}\right) \widetilde{\boldsymbol{E}}\right) \boldsymbol{K}_{s}\left(\boldsymbol{I}-\boldsymbol{\Lambda}_{s}\right) & \boldsymbol{H}_{1}-\boldsymbol{D} \boldsymbol{F}\left(\tau_{k}^{\prime}\right) \widetilde{\boldsymbol{E}} \\ \boldsymbol{\Lambda}_{s} & \boldsymbol{I}-\boldsymbol{\Lambda}_{s} & 0 \\ \boldsymbol{K}_{s} \boldsymbol{\Lambda}_{s} & \boldsymbol{K}_{s}\left(\boldsymbol{I}-\boldsymbol{\Lambda}_{s}\right) & 0\end{array}\right] \\ & =\boldsymbol{H}_{s}+\overline{\boldsymbol{H}} \boldsymbol{K}_{s} \Delta_{s}+\widetilde{\boldsymbol{D}} \boldsymbol{F}\left(\tau_{k}^{\prime}\right) \widetilde{\boldsymbol{E}}\left(\boldsymbol{K}_{s} \Delta_{s}-\overline{\boldsymbol{I}}\right)\end{array}$ and wherein $\boldsymbol{H}_{s}=\left[\begin{array}{ccc}\boldsymbol{G} & 0 & \boldsymbol{H}_{1} \\ \boldsymbol{\Lambda}_{s} & \boldsymbol{I}-\boldsymbol{\Lambda}_{s} & 0 \\ 0 & 0 & 0\end{array}\right], \overline{\boldsymbol{H}}=\left[\begin{array}{c}\boldsymbol{H}_{0} \\ 0 \\ \boldsymbol{I}\end{array}\right], \widetilde{\Delta}_{s}=\left[\boldsymbol{\Lambda}_{s} \boldsymbol{I}-\boldsymbol{\Lambda}_{s} 0\right], \widetilde{\boldsymbol{D}}=\left[\begin{array}{l}\boldsymbol{D} \\ 0 \\ 0\end{array}\right]$, $\overline{\mathbf{I}}=\left[\begin{array}{lll}0 & 0 & \boldsymbol{I}\end{array}\right]$ 


\section{GMRS}

NCS with the MAC and GMRS is depicted in Figure 4. The core of the MRS is that the states that can access the network are dependent on the errors between the ideal dynamic and the physical system. The system without MAC and time delay can be regarded as the ideal dynamic (see [26]). The ideal dynamic and the physical system run in parallel. The errors between the states of the two systems are calculated after the states arrive at the scheduler. Then, the weighted norm of the errors is computed. Finally, the state vector $\hat{x}(k)$, which makes the system performance better, will access the network. Note that the states from the ideal dynamic and physical system are all determined by the same scheduling vector $\delta(k)$ in Figure 4 . In this context, the scheduling vector that makes the system performance better is called as the optimal scheduling in this period.

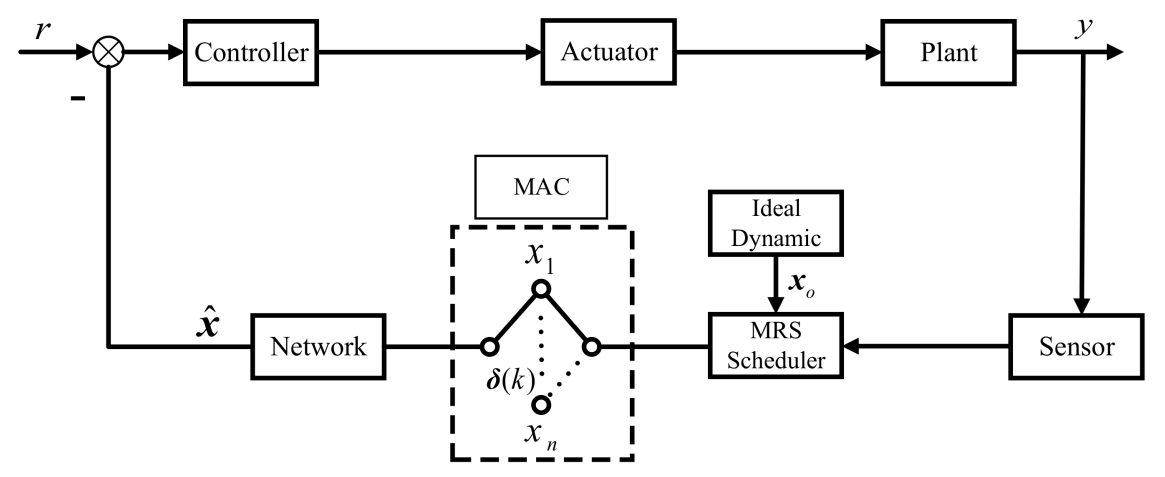

Figure 4. NCS with the GMRS scheme.

$$
\text { Let } \boldsymbol{\xi}(k)=\left[\begin{array}{ll}
x_{o}^{\mathrm{T}}(k) & x^{\mathrm{T}}(k)
\end{array}\right]^{\mathrm{T}}, \boldsymbol{\Pi}=\left[\begin{array}{cc}
\boldsymbol{\Lambda}_{s} & \\
& \boldsymbol{\Lambda}_{s}
\end{array}\right], \boldsymbol{\zeta}=\boldsymbol{\Pi} \boldsymbol{\xi} \text {, then the GMRS scheduler }
$$

can be represented as follows:

$$
S: \rho_{o}=\arg \left(\max _{s \in\{1,2, \ldots N\}}\left\{\rho_{s} \operatorname{norm}\left(\boldsymbol{\zeta}^{\mathrm{T}} \boldsymbol{U} \zeta\right)\right\}\right)
$$

where $\boldsymbol{U}=\operatorname{diag}\left(\boldsymbol{U}_{1},-\boldsymbol{U}_{2}\right)$, and $\boldsymbol{U}_{1}, \boldsymbol{U}_{2}$ are symmetric positive definite matrices. $\arg (\cdot)$ and norm $(\cdot)$ denote the index function and Euclidean norm, respectively. $\rho_{s} \in R$ is a weighted gain on the errors between the states of the ideal dynamic and the states of the physical system.

Remark 1. The states scheduled by the different scheduling vector $\delta(k)$ can be described as $\boldsymbol{\Lambda}_{s} \boldsymbol{x}(k) \in N$, where $\boldsymbol{\Lambda}_{s}$ is the scheduling matrix with its diagonal entities composed of $\boldsymbol{\delta}(k)$, and $N$ is a set named scheduling candidate set. The aim of the GMRS scheduling scheme is to determine the optimal scheduling in the candidate set $N$.

Remark 2. Different from the scheduling scheme in [26], the scheduling condition is extended to a general form in this paper. The introduction of the weighted gain $\rho_{s}$ makes the MDS in [26] a special case of the GMRS. If $\rho_{s}=1$, the GMRS scheme is equivalent to the MDS scheme. The GMRS may be more flexible due to the effect of the weighted gain.

\section{Design of the Guaranteed Performance Controller}

In order to design the guaranteed performance controller, the performance index should be given at first. We define the following quadratic performance index:

$$
J=\sum_{k=0}^{\infty}\left[\boldsymbol{x}^{\mathrm{T}}(k) \boldsymbol{Q} \boldsymbol{x}(k)+\boldsymbol{u}^{\mathrm{T}}(k) \boldsymbol{R} \boldsymbol{u}(k)\right]
$$


where $Q$ and $R$ are given weighted positive definite matrices. Then, the control law of the guaranteed performance can be defined as in Definition 1 .

Definition 1. For systems (1) and (8) and performance index (12), if there exist a control signal $\boldsymbol{u}(k)$ and a positive integer $J^{*}$, such that systems (4), (7), and (10) are asymptotically stable with the performance index $J \leq J^{*}$. We call the control signal $\boldsymbol{u}(k)$ the guaranteed performance control law and $J^{*}$ the guaranteed performance bound.

In order to analyze the system stability and design the controller gain for the NCS with uncertainty and MAC, the following lemmas should be given at first.

Lemma 1 [27]. Consider a symmetric matrix $\boldsymbol{X}$ partitioned into blocks:

$$
X=\left[\begin{array}{cc}
A & B \\
B^{\mathrm{T}} & C
\end{array}\right]
$$

where both $\boldsymbol{A}$ and $\boldsymbol{C}$ are symmetric, square, and non-negative definite, then the following properties are equivalent:

(1) $\boldsymbol{X}$ is negative semi-definite.

(2) The Schur complement of $\boldsymbol{C}$ in $\boldsymbol{X}$, denoted as $\boldsymbol{A}-\boldsymbol{B} \boldsymbol{C}^{-1} \boldsymbol{B}^{\mathrm{T}}$, is negative semi-definite.

(3) The Schur complement of $\boldsymbol{A}$ in $\boldsymbol{X}$, denoted as $\boldsymbol{C}-\boldsymbol{B}^{\mathrm{T}} \boldsymbol{A}^{-1} \boldsymbol{B}$, is negative semi-definite.

Lemma 2 [28]. Suppose that $\boldsymbol{W}, \boldsymbol{M}, \boldsymbol{N}$, and $\boldsymbol{F}(k)$ are real matrices of appropriate dimensions, where $\boldsymbol{F}^{\mathrm{T}}(k) \boldsymbol{F}(k) \leq \boldsymbol{I}$, and $\boldsymbol{W}$ is a symmetric matrix. Then, $\boldsymbol{W}+\operatorname{MF}(k) \boldsymbol{N}+\boldsymbol{N}^{\mathrm{T}} \boldsymbol{F}^{\mathrm{T}}(k) \boldsymbol{M}^{\mathrm{T}}<0$, if and only if there is a constant $\varepsilon>0$ that makes the following inequation hold.

$$
\boldsymbol{W}+\varepsilon \boldsymbol{M} \boldsymbol{M}^{\mathrm{T}}+\varepsilon^{-1} \boldsymbol{N}^{\mathrm{T}} \boldsymbol{N}<0
$$

\subsection{Controller with Uncertainty in the System Matrix and without Zero-Order Hold}

Theorem 1. For the NCS (4) with uncertainty in the system matrix, MAC without zero-order hold, and the performance index (12), the NCS is asymptotically stable with the control law $\boldsymbol{u}(k)$ and performance index bound $J^{*}$, if the following condition holds:

$$
\boldsymbol{\Phi}_{s}^{\mathrm{T}} \boldsymbol{P} \boldsymbol{\Phi}_{s}-\boldsymbol{P}+\boldsymbol{Q}+\boldsymbol{\Lambda}_{s}^{\mathrm{T}} \boldsymbol{K}_{s}^{\mathrm{T}} \boldsymbol{R} \boldsymbol{K}_{s} \boldsymbol{\Lambda}_{s}<0
$$

Proof. The proof is presented in Appendix A.

Theorem 2. The NCS (4) with uncertainty in the system matrix and MAC without zero-order hold is asymptotically stable, if there exist an asymmetric positive definite matrix $X \in R^{n \times n}$ and a scalar $\varepsilon>0$, such that:

$$
\left[\begin{array}{ccccc}
-\boldsymbol{X} & * & * & * & * \\
\mathrm{AX}+\boldsymbol{B} \boldsymbol{Y}_{s} & -\boldsymbol{X}+\varepsilon \boldsymbol{D} \boldsymbol{D}^{\mathrm{T}} & * & * & * \\
\mathrm{EX} & 0 & -\varepsilon \boldsymbol{I} & * & * \\
\boldsymbol{Y}_{s} & 0 & 0 & -\boldsymbol{R}^{-1} & * \\
\boldsymbol{X} & 0 & 0 & 0 & -\boldsymbol{Q}^{-1}
\end{array}\right]<0
$$

The guaranteed performance controller gain can be calculated by $K_{s}=\boldsymbol{Y}_{s} \boldsymbol{X}^{-1}\left(\boldsymbol{\Lambda}_{s}\right)^{+}$, where $\left(\boldsymbol{\Lambda}_{s}\right)^{+}$means a generalized inverse of the matrix $\boldsymbol{\Lambda}_{s}$. 
Proof. The proof is presented in Appendix B.

\subsection{Controller with Uncertainty in the System Matrix and with Zero-Order Hold}

For the NCS (7), the guaranteed performance index (12) should be rewritten as the following form.

$$
J=\sum_{k=0}^{\infty}\left[x^{T}(k) \boldsymbol{Q x}(k)+u^{T}(k) \boldsymbol{R} \boldsymbol{u}(k)\right]=\sum_{\infty}^{k=0} z^{T}(k) \Psi z(k)
$$

where $\boldsymbol{\Psi}=\overline{\boldsymbol{Q}}+\overline{\boldsymbol{R}}$, and $\overline{\boldsymbol{Q}}=\left[\begin{array}{cc}\boldsymbol{Q} & 0 \\ 0 & 0\end{array}\right]=\hat{\boldsymbol{E}}^{\mathrm{T}} \boldsymbol{Q} \hat{\boldsymbol{E}}, \hat{\boldsymbol{E}}=\left[\begin{array}{ll}\boldsymbol{I} & 0\end{array}\right], \overline{\boldsymbol{R}}=\Delta_{s}^{\mathrm{T}} \boldsymbol{K}_{s}^{\mathrm{T}} \boldsymbol{R} \boldsymbol{K}_{s} \Delta_{s}$.

Theorem 3. For the NCS (7) with uncertainty in the system matrix, MAC with zero-order hold, and the performance index (12), the NCS is asymptotically stable with the control law $\boldsymbol{u}(k)$ and performance index bound $J^{*}$, if the following inequation holds:

$$
\boldsymbol{\Theta}_{s}^{\mathrm{T}} \mathbf{P} \boldsymbol{\Theta}_{s}-\boldsymbol{P}+\overline{\boldsymbol{Q}}+\overline{\boldsymbol{R}}<0
$$

Proof. The proof is presented in Appendix C.

Theorem 4. The NCS (7) with uncertainty in the system matrix and MAC with zero-order hold is asymptotically stable, if there exist an asymmetric positive definite matrix $X \in R^{n \times n}$ and a scalar $\varepsilon>0$, such that:

$$
\left[\begin{array}{ccccc}
-\boldsymbol{X} & * & * & * & * \\
\overline{\boldsymbol{A}}_{S} \boldsymbol{X}+\overline{\boldsymbol{B}} \boldsymbol{Y}_{S} & -\boldsymbol{X}+\varepsilon \overline{\boldsymbol{D}}^{\mathrm{T}} & * & * & * \\
\overline{\boldsymbol{E}} \boldsymbol{X} & 0 & -\varepsilon \boldsymbol{I} & * & * \\
\boldsymbol{Y}_{S} & 0 & 0 & -\boldsymbol{R}^{-1} & * \\
\hat{\boldsymbol{E} X} & 0 & 0 & 0 & -\boldsymbol{Q}^{-1}
\end{array}\right]<0
$$

The guaranteed performance controller gain can be calculated by $K_{s}=Y_{s} X^{-1}\left(\Delta_{s}\right)^{+}$, where $\left(\Delta_{s}\right)^{+}$is a generalized inverse of the matrix $\Delta_{s}$.

Proof. The proof is presented in Appendix D.

\subsection{Controller with Uncertainty in the Control Matrix}

For the NCS (10), the guaranteed performance index (12) should be replaced by the following form:

$$
J=\sum_{k=0}^{\infty}\left[x^{\mathrm{T}}(k) \boldsymbol{Q x}(k)+\boldsymbol{u}^{\mathrm{T}}(k) \boldsymbol{R} \boldsymbol{u}(k)\right]=\sum_{\infty}^{k=0} \boldsymbol{v}^{\mathrm{T}}(k) \boldsymbol{\Xi} \boldsymbol{v}(k)
$$

where $\boldsymbol{\Xi}=\widetilde{\boldsymbol{Q}}+\widetilde{\boldsymbol{R}}$, and $\widetilde{\boldsymbol{Q}}=\left[\begin{array}{ccc}\boldsymbol{Q} & 0 & 0 \\ 0 & 0 & 0 \\ 0 & 0 & 0\end{array}\right]=\widehat{\boldsymbol{E}} \widehat{\mathrm{Q}} \widehat{\boldsymbol{E}}, \widehat{\boldsymbol{E}}=\left[\begin{array}{lll}\boldsymbol{I} & 0 & 0\end{array}\right], \widetilde{\boldsymbol{R}}=\widetilde{\Delta}_{s}^{\mathrm{T}} \boldsymbol{K}_{s}^{\mathrm{T}} \boldsymbol{R} \boldsymbol{K}_{s} \widetilde{\Delta}_{s}$.

Theorem 5. For the NCS (10) with uncertainty in the control matrix, MAC with zero-order hold, and the performance index (12), the NCS is asymptotically stable with the control law $\boldsymbol{u}(k)$ and performance index bound $J^{*}$, if the following inequation holds:

$$
\boldsymbol{\Omega}_{s}^{\mathrm{T}} \boldsymbol{P} \boldsymbol{\Omega}_{s}-\boldsymbol{P}+\widetilde{\boldsymbol{Q}}+\widetilde{\boldsymbol{R}}<0
$$


Proof. The proof is presented in Appendix E.

Theorem 6. The NCS (10) with uncertainty in the control matrix and MAC with zero-order hold is asymptotically stable, if there exist an asymmetric positive definite matrix $X \in R^{n \times n}$ and a scalar $\varepsilon>0$, such that:

$$
\left[\begin{array}{ccccc}
-\boldsymbol{X} & * & * & * & * \\
\boldsymbol{H}_{s} \boldsymbol{X}+\overline{\boldsymbol{H}} \boldsymbol{Y}_{S} & -\boldsymbol{X}+\varepsilon \widetilde{\boldsymbol{D}} \widetilde{\boldsymbol{D}}^{\mathrm{T}} & * & * & * \\
\widetilde{\boldsymbol{E}} \boldsymbol{Y}_{S}-\widetilde{\boldsymbol{E}} \overline{\boldsymbol{I}} \boldsymbol{X} & 0 & -\varepsilon \boldsymbol{I} & * & * \\
\boldsymbol{Y}_{S} & 0 & 0 & -\boldsymbol{R}^{-1} & * \\
\widehat{\boldsymbol{E} X} & 0 & 0 & 0 & -\boldsymbol{Q}^{-1}
\end{array}\right]<0
$$

The guaranteed performance controller gain can be calculated by $K_{s}=\boldsymbol{Y}_{s} \boldsymbol{X}^{-1}\left(\widetilde{\Delta}_{s}\right)^{+}$, where $\left(\widetilde{\Delta}_{s}\right)^{+}$is a generalized inverse of the matrix $\widetilde{\Delta}_{s}$.

Proof. The proof is presented in Appendix F.

Remark 3. Note that the controller design of the ideal dynamic is similar to the one in [26], and it is omitted here due to the limited space. It can be further noted that the trajectory of the ideal dynamic provides a bound on the states of the physical system as the negative factors from the network and the uncertainty of the modeling are eliminated. The states that can access the network just depends on their weighted norm of the errors between the ideal dynamic and the physical system according to the principle of the GMRS.

\section{Illustrative Examples}

To demonstrate the effectiveness of the co-design scheme proposed in the paper, some cases of simulation are given in terms of the uncertainty in the system matrix without zeroorder hold, the uncertainty in the system matrix with zero-order hold, and the uncertainty in the control matrix, respectively.

Case 1. Effectiveness of the co-design of the guaranteed performance control and the GMRS without zero-order hold for the NCS with the uncertainty in the system matrix. The calculation of the parameters of the ideal dynamic can be referred to the work in [26].

Consider the following system parameters for the system (1):

$$
A=\left[\begin{array}{cc}
0.98 & 0 \\
0 & 0.15
\end{array}\right], B=\left[\begin{array}{l}
1 \\
2
\end{array}\right]
$$

Assume that only one of the states can gain access to the network in each sampling instant. In this context, two scheduling vectors are generated, i.e., $\delta_{1}(k)=\left[\begin{array}{ll}1 & 0\end{array}\right]$ and $\delta_{2}(k)=\left[\begin{array}{ll}0 & 1\end{array}\right]$. Without loss of generality, let $\boldsymbol{U}_{1}=\boldsymbol{U}_{2}=\left[\begin{array}{ll}1 & \\ & 1\end{array}\right]$ in (11). We set $\boldsymbol{D}=\left[\begin{array}{cc}0.1 & 0 \\ 0 & 0.1\end{array}\right], \boldsymbol{E}=\left[\begin{array}{cc}0.2 & 0.3 \\ 0.1 & 0.4\end{array}\right], \boldsymbol{Q}=\left[\begin{array}{cc}0.5 & 0 \\ 0 & 0.5\end{array}\right]$, and $\boldsymbol{R}=1$. The performance of the co-design of the control and GMRS for the NCS without zero-order hold is shown in Figure 5, while the scheduling of the co-design of the control and GMRS is shown in Figure 6. 


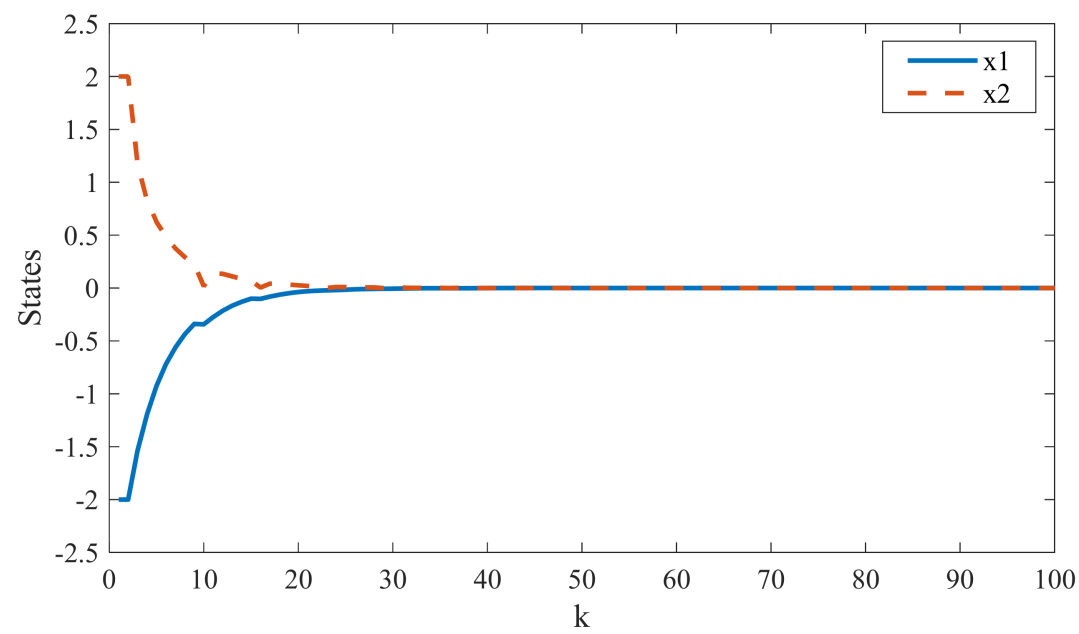

Figure 5. Performance of the co-design of the control and GMRS for the NCS without zero-order hold.

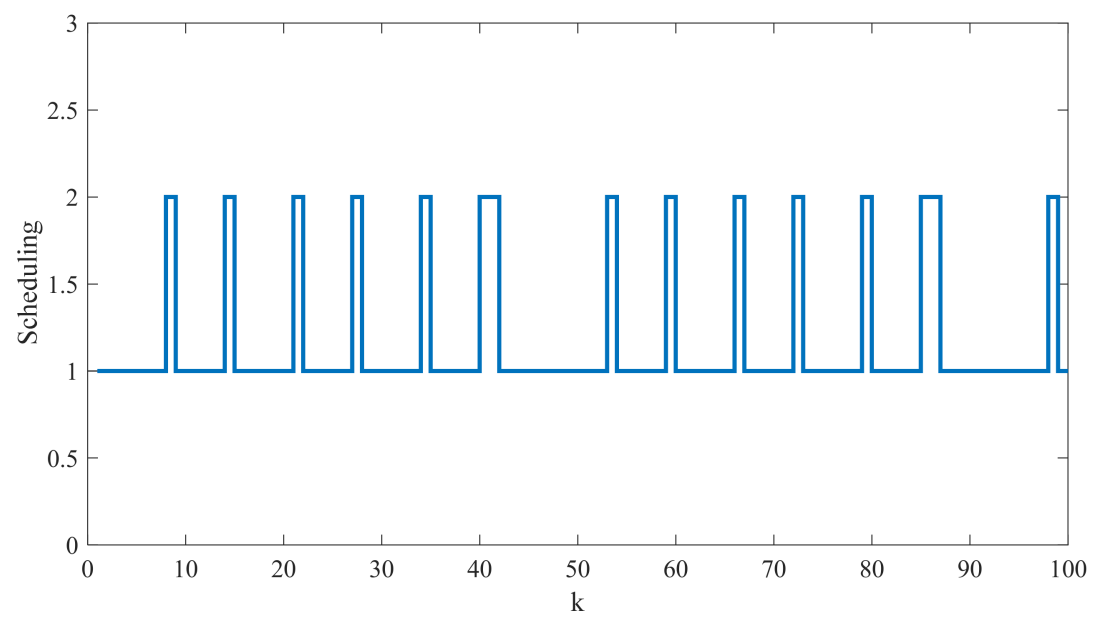

Figure 6. Scheduling of the co-design of the control and GMRS for the NCS without zero-order hold.

From Figure 5, the states of the given NCS without zero-order hold converge to the equilibrium at $k=18$. It is found that the co-design of the control and GMRS is effective for the NCS without zero-order hold. In Figure 6, ' 1 ' on the ordinate means the first state gains the access to the network while ' 2 ' means the other state gains the access to the network. From the angle of the scheduling, as shown in Figure 6, the scheduler switches limit times. This causes a great reduction in the load of the scheduler.

Case 2. Effectiveness of the co-design of the guaranteed performance control and the GMRS with zero-order hold for the NCS with the uncertainty in the system matrix.

The system parameters are adopted the same as in Case 1 in order to make an easy comparison between the systems with and without zero-order hold. The performance of the co-design of the control and GMRS for the NCS with zero-order hold is shown in Figure 7, while the scheduling of the co-design of the control and GMRS is shown in Figure 8. 


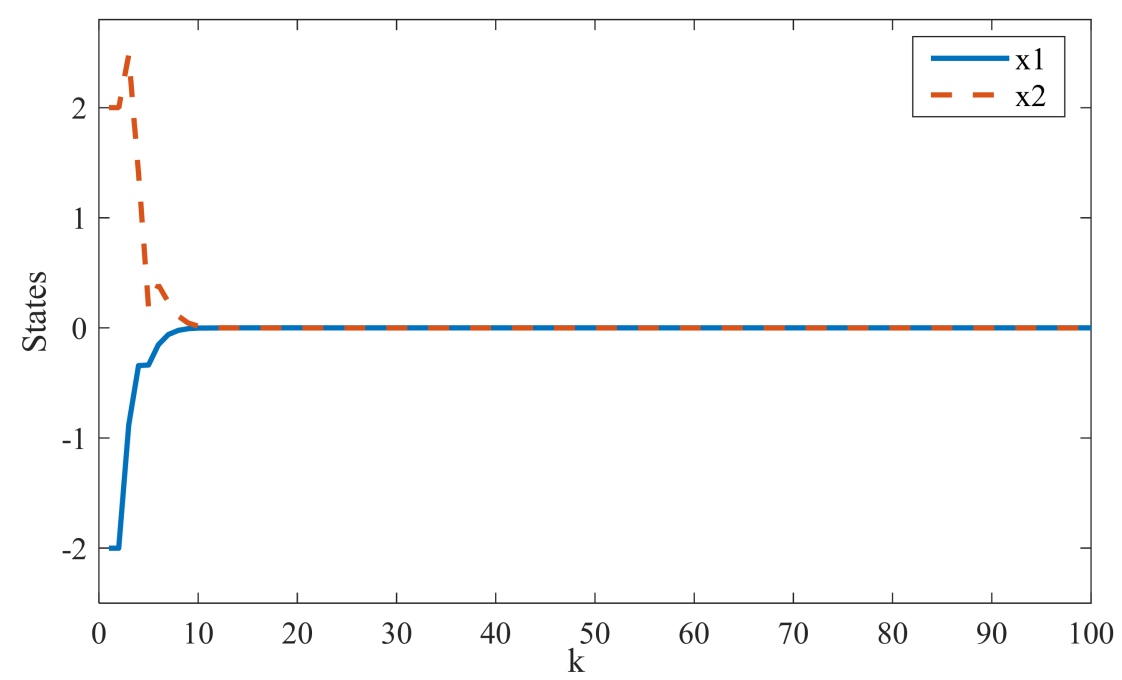

Figure 7. Performance of the co-design of the control and GMRS for the NCS with zero-order hold.

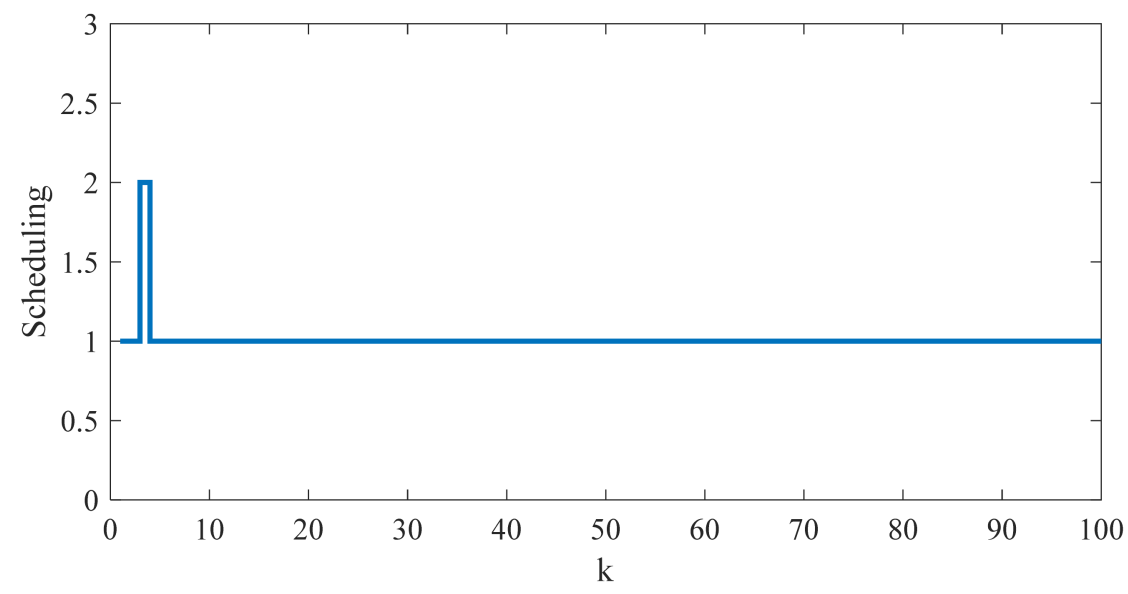

Figure 8. Scheduling of the co-design of the control and GMRS for the NCS with zero-order hold.

The states of the given NCS with zero-order hold converge to the equilibrium at $k=10$. It means that the co-design of the control and GMRS is more effective for the NCS with the zero-order hold than the one without the zero-order hold. On the other hand, as shown in Figure 8, the scheduler switches fewer times in comparison with the scheduling in Figure 6.

Remark 4. From the perspective of the system performance, the system performance with the zero-order hold is better than that of the one without the zero-order hold. However, the design of the former is more complex, and it may use more computing resources. On the other hand, the load of the scheduler with the zero-order hold is smaller than that of the one without the zero-order hold. We can see that the system performance is improved at the cost of rising system complexity and consuming more computing resources. Therefore, we can choose a zero-order hold or not based on the demand of the system performance and resources.

Case 3. Effectiveness of the co-design of the guaranteed performance control and the GMRS for the NCS with the uncertainty in the control matrix.

Consider a continuous control system with $A=\left[\begin{array}{cc}-1 & 0 \\ 3 & -4\end{array}\right], \boldsymbol{B}=\left[\begin{array}{c}-1 \\ 2\end{array}\right]$ in (8), the sampling period is assumed to be $T=0.4 \mathrm{~s}$, and the random time delay satisfies $\tau_{k}<T$. We let $\rho_{1}=100$ and $\rho_{2}=1$.

In order to demonstrate the effectiveness of the proposed scheduling and the codesign method, the performance of the GMRS is compared with the MDS in [26] and 
MEF-TOD in [20]. Seen from Figure 9, the NCS with MEF-TOD scheduling converges to the equilibrium at around $8 \mathrm{~s}$, and the one with MDS converges to the equilibrium at around $7 \mathrm{~s}$. However, the one with the GMRS only needs about $6 \mathrm{~s}$. Hence, the stability performance of the GMRS is better than that of the MEF-TOD scheduling and MDS scheme for the NCS with the uncertainty in the control matrix.

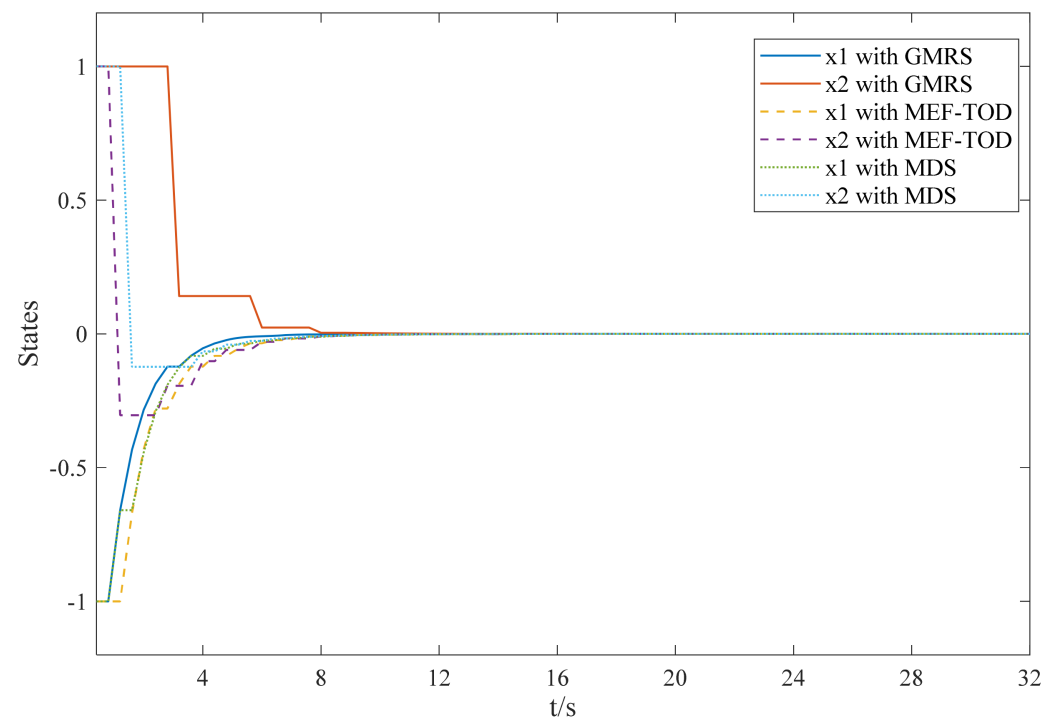

Figure 9. System performance comparisons.

\section{Conclusions}

NCS is a research focus on the control, communication, and computer fields. Many challenges from the introduction of networks, such as time delay, data packet dropout, quantization, and data packet disorder, should be tackled comprehensively. As an important concern in NCS, guaranteed performance control has attracted more and more attention. Modeling uncertainty and the uncertainty from the introduction of the network, such as time delay, should be studied in-depth.

In this paper, we propose a GMRS scheduling scheme, and construct a co-design of the GMRS and guaranteed performance control. In order to evaluate the effectiveness of the proposed scheduling approach, comparisons with the widely used MEF-TOD strategy proposed in [20] and the MDS scheme proposed in [26] are given. The performance of the GMRS is relatively better than the existing two scheduling strategies. It can be further noted that the introduction of the ideal dynamic improves the performance of the MEF-TOD scheduling scheme. Meanwhile, the introduction of the weighted gain parameter can relax the conservatism of the MDS scheme, and the control performance is obviously improved. In addition, it is illustrated that the zero-order hold mechanism is more efficient for the NCS with the uncertainty in the system matrix.

Future work will be devoted to an extension of the GMRS toward consideration of other network imperfections, such as packet dropouts, quantization, and packet disorder effects. Meanwhile, more application examples based on the experimental setup should be studied with the GMRS.

Author Contributions: Conceptualization, S.Z. and C.Z.; methodology, S.Z. and L.S.; validation, S.Z., C.Z. and L.S.; formal analysis, S.Z.; investigation, S.Z. and C.Z.; writing-original draft preparation, S.Z.; writing-review and editing, S.Z., C.Z. and L.S. All authors have read and agreed to the published version of the manuscript.

Funding: This research was funded by the Natural Science Foundation of Tianjin (grant number 18JCQNJC74600) and the Open Fund of Tianjin Key Laboratory for Control Theory \& Applications in Complicated System (grant numbers TJKL-CTACS-201704 and TJKL-CTACS-201807). 
Acknowledgments: The authors would like to thank the editors and anonymous reviewers for their useful comments for improving the quality of this paper.

Conflicts of Interest: The authors declare no conflict of interest.

\section{Abbreviations}

The following abbreviations are used in this manuscript:

NCS Networked Control System

T-S Takagi-Sugeno

MIMO Multiple Input Multiple Output

ETM Event-triggering Mechanism

MAC Medium Access Constraint

RM Rate Monotonic

EDF Earliest Deadline First

MEF-TOD Maximum Error First and Try Once Discard

MRS Model Reference Scheduling

GMRS Generalized Model-reference Scheduling

MDS Model-dependent Scheduling

\section{Appendix A. Proof of Theorem 1}

Proof. We choose the following Lyapunov function:

$$
V(\boldsymbol{x}(k))=\boldsymbol{x}^{\mathrm{T}}(k) \boldsymbol{P} \boldsymbol{x}(k)
$$

Then, $\Delta V(x(k))=V(x(k+1))-V(x(k))=x^{\mathrm{T}}(k) \boldsymbol{\Phi}_{s}^{\mathrm{T}} \boldsymbol{P} \boldsymbol{\Phi}_{s} x(k)-x^{\mathrm{T}}(k) \boldsymbol{P} \boldsymbol{x}(k)$ According to inequation (13), we have:

$$
\boldsymbol{x}^{\mathrm{T}}(k) \boldsymbol{\Phi}_{s}^{\mathrm{T}} \boldsymbol{P} \boldsymbol{\Phi}_{s} \boldsymbol{x}(k)-\boldsymbol{x}^{\mathrm{T}}(k) \boldsymbol{P} \boldsymbol{x}(k)+\boldsymbol{x}^{\mathrm{T}}(k) \boldsymbol{Q} \boldsymbol{x}(k)+\boldsymbol{x}^{\mathrm{T}}(k) \boldsymbol{\Lambda}_{s}^{\mathrm{T}} \boldsymbol{K}_{s}^{\mathrm{T}} \boldsymbol{R} \boldsymbol{K}_{s} \boldsymbol{\Lambda}_{s} \boldsymbol{x}(k)<0
$$

That is,

$$
\boldsymbol{x}^{\mathrm{T}}(k) \boldsymbol{\Phi}_{s}^{\mathrm{T}} \boldsymbol{P} \boldsymbol{\Phi}_{s} \boldsymbol{x}(k)-\boldsymbol{x}^{\mathrm{T}}(k) \boldsymbol{P} \boldsymbol{x}(k)+\boldsymbol{x}^{\mathrm{T}}(k) \boldsymbol{Q x}(k)+\boldsymbol{u}^{\mathrm{T}}(k) \boldsymbol{R} \boldsymbol{u}(k)<0
$$

This means that:

$$
\Delta V(x(k))<-x^{\mathrm{T}}(k) \boldsymbol{Q x}(k)-\boldsymbol{u}^{\mathrm{T}}(k) \boldsymbol{R} \boldsymbol{u}(k)<0
$$

The proof is completed.

\section{Appendix B. Proof of Theorem 2}

Proof. Using Lemma 1, inequation (13) can be written as:

$$
\left[\begin{array}{cc}
-\boldsymbol{P}+\boldsymbol{Q}+\boldsymbol{\Lambda}_{s}^{\mathrm{T}} \boldsymbol{K}_{s}^{\mathrm{T}} \boldsymbol{R} \boldsymbol{K}_{s} \boldsymbol{\Lambda}_{s} & * \\
\boldsymbol{\Phi}_{s} & -\boldsymbol{P}^{-1}
\end{array}\right]<0
$$

Due to $\boldsymbol{\Phi}_{s}=A+\Delta A+B K_{s} \Lambda_{s}$, then the following inequation holds.

$$
\left[\begin{array}{cc}
-\boldsymbol{P}+\boldsymbol{Q}+\boldsymbol{\Lambda}_{s}^{\mathrm{T}} \boldsymbol{K}_{s}^{\mathrm{T}} \boldsymbol{R} \boldsymbol{K}_{s} \boldsymbol{\Lambda}_{s} & * \\
\boldsymbol{A}+\Delta \boldsymbol{A}+\boldsymbol{B} \boldsymbol{K}_{s} \boldsymbol{\Lambda}_{s} & -\boldsymbol{P}^{-1}
\end{array}\right]<0
$$

Substituting $\triangle A=D F E$ into (A6), we have:

$$
\left[\begin{array}{cc}
-\boldsymbol{P}+\boldsymbol{Q}+\boldsymbol{\Lambda}_{s}^{\mathrm{T}} \boldsymbol{K}_{s}^{\mathrm{T}} \boldsymbol{R} \boldsymbol{K}_{s} \boldsymbol{\Lambda}_{s} & * \\
\boldsymbol{A}+\boldsymbol{B} \boldsymbol{K}_{s} \boldsymbol{\Lambda}_{s} & -\boldsymbol{P}^{-1}
\end{array}\right]+\left[\begin{array}{c}
0 \\
\boldsymbol{D}
\end{array}\right] \boldsymbol{F}\left[\begin{array}{ll}
\boldsymbol{E} & 0
\end{array}\right]+\left[\begin{array}{ll}
\boldsymbol{E} & 0
\end{array}\right]^{\mathrm{T}} \boldsymbol{F}^{\mathrm{T}}\left[\begin{array}{c}
0 \\
\boldsymbol{D}
\end{array}\right]^{\mathrm{T}}<0
$$


From Lemma 2, (A7) can be rewritten as:

$$
\left[\begin{array}{cc}
-\boldsymbol{P}+\boldsymbol{Q}+\boldsymbol{\Lambda}_{s}^{\mathrm{T}} \boldsymbol{K}_{s}^{\mathrm{T}} \boldsymbol{R} \boldsymbol{K}_{s} \boldsymbol{\Lambda}_{s} & * \\
\boldsymbol{A}+\boldsymbol{B} \boldsymbol{K}_{s} \boldsymbol{\Lambda}_{s} & -\boldsymbol{P}^{-1}
\end{array}\right]+\varepsilon\left[\begin{array}{c}
0 \\
\boldsymbol{D}
\end{array}\right]\left[\begin{array}{c}
0 \\
\boldsymbol{D}
\end{array}\right]^{\mathrm{T}}+\varepsilon^{-1}\left[\begin{array}{ll}
\boldsymbol{E} & 0
\end{array}\right]^{\mathrm{T}}\left[\begin{array}{ll}
\boldsymbol{E} & 0
\end{array}\right]<0
$$

According to Lemma 1, we obtain the following inequation:

$$
\left[\begin{array}{cccc}
-\boldsymbol{P}+\boldsymbol{Q} & * & * & * \\
\boldsymbol{A}+\boldsymbol{B} \boldsymbol{K}_{s} \boldsymbol{\Lambda}_{s} & -\boldsymbol{P}^{-1}+\varepsilon \boldsymbol{D} \boldsymbol{D}^{\mathrm{T}} & * & * \\
\boldsymbol{E} & 0 & -\varepsilon \boldsymbol{I} & * \\
\boldsymbol{K}_{s} \boldsymbol{\Lambda}_{s} & 0 & 0 & -\boldsymbol{R}^{-1}
\end{array}\right]<0
$$

Note that inequation (A9) is not a feasible LMI. In order to obtain a feasible LMI, (A9) is pre-multiplied and post-multiplied by $\operatorname{diag}\left(\left[\begin{array}{llll}\boldsymbol{P}^{-1} & \boldsymbol{I} & \boldsymbol{I} & \boldsymbol{I}\end{array}\right]\right)$. Let $\boldsymbol{P}^{-1}=\boldsymbol{X}, \boldsymbol{Y}_{S}=\boldsymbol{K}_{s} \boldsymbol{\Lambda}_{s} \boldsymbol{X}$, then we have:

$$
\left[\begin{array}{cccc}
-\boldsymbol{X}+\boldsymbol{X} \boldsymbol{Q X} & * & * & * \\
\boldsymbol{A X}+\boldsymbol{B} \boldsymbol{Y}_{S} & -\boldsymbol{X}+\varepsilon \boldsymbol{D} \boldsymbol{D}^{\mathrm{T}} & * & * \\
\boldsymbol{E X} & 0 & -\varepsilon \boldsymbol{I} & * \\
\boldsymbol{Y}_{S} & 0 & 0 & -\boldsymbol{R}^{-1}
\end{array}\right]<0
$$

According to Lemma 1, inequation (14) holds. The proof is completed.

\section{Appendix C. Proof of Theorem 3}

Proof. We choose the following Lyapunov function:

$$
V(z(k))=z^{\mathrm{T}}(k) \boldsymbol{P} z(k)
$$

Then, $\Delta V(z(k))=V(z(k+1))-V(z(k))=\boldsymbol{z}^{\mathrm{T}}(k) \boldsymbol{\Theta}_{S}^{\mathrm{T}} \boldsymbol{P} \boldsymbol{\Theta}_{s} \boldsymbol{z}(k)-\boldsymbol{z}^{\mathrm{T}}(k) \boldsymbol{P} \boldsymbol{z}(k)$ According to inequation (16), we have:

$$
\boldsymbol{z}^{\mathrm{T}}(k) \boldsymbol{\Theta}_{s}^{\mathrm{T}} \boldsymbol{P} \boldsymbol{\Theta}_{s} z(k)-\boldsymbol{z}^{\mathrm{T}}(k) \boldsymbol{P} \boldsymbol{z}(k)+\boldsymbol{z}^{\mathrm{T}}(k) \overline{\boldsymbol{Q}} \boldsymbol{z}(k)+\boldsymbol{z}^{\mathrm{T}}(k) \overline{\boldsymbol{R}} \boldsymbol{z}(k)<0
$$

That is,

$$
\Delta V(z(k))<-z^{\mathrm{T}}(k) \boldsymbol{\Psi} z(k)<0
$$

The proof is completed.

\section{Appendix D. Proof of Theorem 4}

Proof. Using Lemma 1, inequation (16) can be written as follows:

$$
\left[\begin{array}{cc}
-\boldsymbol{P}+\overline{\boldsymbol{Q}}+\Delta_{S}^{\mathrm{T}} \boldsymbol{K}_{s}^{\mathrm{T}} \boldsymbol{R} \boldsymbol{K}_{s} \Delta_{S} & * \\
\boldsymbol{\Theta}_{S} & -\boldsymbol{P}^{-1}
\end{array}\right]<0
$$

Due to $\Theta_{s}=\bar{A}_{s}+\bar{D} F \bar{E}+\bar{B} K_{s} \Delta_{s}$, then the following inequation holds.

$$
\left[\begin{array}{cc}
-\boldsymbol{P}+\overline{\boldsymbol{Q}}+\Delta_{s}^{\mathrm{T}} \boldsymbol{K}_{s}^{\mathrm{T}} \boldsymbol{R} \boldsymbol{K}_{s} \Delta_{s} & * \\
\overline{\boldsymbol{A}}_{s}+\overline{\boldsymbol{D}} \boldsymbol{F} \overline{\boldsymbol{E}}+\overline{\boldsymbol{B}} \boldsymbol{K}_{s} \Delta_{s} & -\boldsymbol{P}^{-1}
\end{array}\right]<0
$$

Inequation (A15) can be rewritten as:

$$
\left[\begin{array}{cc}
-\boldsymbol{P}+\overline{\boldsymbol{Q}}+\Delta_{s}^{\mathrm{T}} \boldsymbol{K}_{s}^{\mathrm{T}} \boldsymbol{R} \boldsymbol{K}_{s} \Delta_{s} & * \\
\overline{\boldsymbol{A}}_{s}+\overline{\boldsymbol{B}} \boldsymbol{K}_{s} \Delta_{s} & -\boldsymbol{P}^{-1}
\end{array}\right]+\left[\begin{array}{c}
0 \\
\overline{\boldsymbol{D}}
\end{array}\right] \boldsymbol{F}\left[\begin{array}{cc}
\overline{\boldsymbol{E}} & 0
\end{array}\right]+\left[\begin{array}{ll}
\overline{\boldsymbol{E}} & 0
\end{array}\right]^{\mathrm{T}} \boldsymbol{F}^{\mathrm{T}}\left[\begin{array}{c}
0 \\
\overline{\boldsymbol{D}}
\end{array}\right]^{\mathrm{T}}<0
$$


From Lemma 2, the following inequation is obtained:

$$
\left[\begin{array}{cc}
-\boldsymbol{P}+\overline{\boldsymbol{Q}}+\Delta_{s}^{\mathrm{T}} \boldsymbol{K}_{s}^{\mathrm{T}} \boldsymbol{R} \boldsymbol{K}_{s} \Delta_{s} & * \\
\overline{\boldsymbol{A}}_{s}+\overline{\boldsymbol{B}} \boldsymbol{K}_{s} \Delta_{s} & -\boldsymbol{P}^{-1}
\end{array}\right]+\varepsilon\left[\begin{array}{c}
0 \\
\overline{\boldsymbol{D}}
\end{array}\right]\left[\begin{array}{c}
0 \\
\overline{\boldsymbol{D}}
\end{array}\right]^{\mathrm{T}}+\varepsilon^{-1}\left[\begin{array}{cc}
\overline{\boldsymbol{E}} & 0
\end{array}\right]^{\mathrm{T}}\left[\begin{array}{ll}
\overline{\boldsymbol{E}} & 0]<0
\end{array}\right.
$$

Using Lemma 1 again, the following inequation is attained:

$$
\left[\begin{array}{cccc}
-\boldsymbol{P}+\overline{\boldsymbol{Q}} & * & * & * \\
\overline{\boldsymbol{A}}_{s}+\overline{\boldsymbol{B}} \boldsymbol{K}_{s} \Delta_{s} & -\boldsymbol{P}^{-1}+\varepsilon \overline{\boldsymbol{D D}}^{\mathrm{T}} & * & * \\
\overline{\boldsymbol{E}} & 0 & -\varepsilon \boldsymbol{I} & * \\
\boldsymbol{K}_{s} \Delta_{s} & 0 & 0 & -\boldsymbol{R}^{-1}
\end{array}\right]<0
$$

Note that inequation (A18) is not an LMI. In order to obtain a feasible LMI, (A18) is pre-multiplied and post-multiplied by $\operatorname{diag}\left(\left[\begin{array}{llll}\boldsymbol{P}^{-1} & \boldsymbol{I} & \boldsymbol{I} & \boldsymbol{I}\end{array}\right]\right)$. Let $\boldsymbol{P}^{-1}=\boldsymbol{X}, \boldsymbol{Y}_{S}=\boldsymbol{K}_{s} \Delta_{S} \boldsymbol{X}$; then, we have

$$
\left[\begin{array}{cccc}
-\boldsymbol{X}+\boldsymbol{X} \overline{\mathbf{Q}} \boldsymbol{X} & * & * & * \\
\overline{\boldsymbol{A}}_{S} \boldsymbol{X}+\overline{\boldsymbol{B}} \boldsymbol{Y}_{S} & -\boldsymbol{X}+\varepsilon \overline{\boldsymbol{D D}}^{\mathrm{T}} & * & * \\
\overline{\boldsymbol{E}} \boldsymbol{X} & 0 & -\varepsilon \boldsymbol{I} & * \\
\boldsymbol{Y}_{S} & 0 & 0 & -\boldsymbol{R}^{-1}
\end{array}\right]<0
$$

According to Lemma 1, inequation (17) holds. The proof is completed.

\section{Appendix E. Proof of Theorem 5}

Proof. Choosing the following Lyapunov function,

$$
V(\boldsymbol{v}(k))=\boldsymbol{v}^{\mathrm{T}}(k) \boldsymbol{P} \boldsymbol{v}(k)
$$

then, $\Delta V(\boldsymbol{v}(k))=V(\boldsymbol{v}(k+1))-V(\boldsymbol{v}(k))=\boldsymbol{v}^{\mathrm{T}}(k) \boldsymbol{\Omega}_{S}^{\mathrm{T}} \boldsymbol{P} \boldsymbol{\Omega}_{s} \boldsymbol{v}(k)-\boldsymbol{v}^{\mathrm{T}}(k) \boldsymbol{P} \boldsymbol{v}(k)$

According to inequation (19), we have:

$$
\boldsymbol{v}^{\mathrm{T}}(k) \boldsymbol{\Omega}_{s}^{\mathrm{T}} \boldsymbol{P} \boldsymbol{\Omega}_{s} \boldsymbol{v}(k)-\boldsymbol{v}^{\mathrm{T}}(k) \boldsymbol{P} \boldsymbol{v}(k)+\boldsymbol{v}^{\mathrm{T}}(k) \widetilde{\boldsymbol{Q}} \boldsymbol{v}(k)+\boldsymbol{v}^{\mathrm{T}}(k) \widetilde{\boldsymbol{R}} \boldsymbol{v}(k)<0
$$

That is,

$$
\Delta V(\boldsymbol{v}(k))<-\boldsymbol{v}^{\mathrm{T}}(k) \boldsymbol{\Psi} \boldsymbol{v}(k)<0
$$

The proof is completed.

\section{Appendix F. Proof of Theorem 6}

Proof. Using Lemma 1, inequation (19) can be written as follows:

$$
\left[\begin{array}{cc}
-\boldsymbol{P}+\widetilde{\boldsymbol{Q}}+\widetilde{\Delta}_{s}^{\mathrm{T}} \boldsymbol{K}_{s}^{\mathrm{T}} \boldsymbol{R} \boldsymbol{K}_{s} \widetilde{\Delta}_{s} & * \\
\boldsymbol{\Omega}_{S} & -\boldsymbol{P}^{-1}
\end{array}\right]<0
$$

Due to $\boldsymbol{\Omega}_{s}=\boldsymbol{H}_{S}+\overline{\boldsymbol{H}} \boldsymbol{K}_{s} \Delta_{s}+\widetilde{\boldsymbol{D}} \boldsymbol{F}\left(\tau_{k}^{\prime}\right) \widetilde{\boldsymbol{E}}\left(\boldsymbol{K}_{s} \Delta_{s}-\overline{\boldsymbol{I}}\right)$, the following inequation holds:

$$
\left[\begin{array}{cc}
-\boldsymbol{P}+\widetilde{\boldsymbol{Q}}+\widetilde{\Delta}_{s}^{\mathrm{T}} \boldsymbol{K}_{s}^{\mathrm{T}} \boldsymbol{R} \boldsymbol{K}_{s} \widetilde{\Delta}_{s} & * \\
\boldsymbol{H}_{s}+\overline{\boldsymbol{H}} \boldsymbol{K}_{s} \Delta_{s}+\widetilde{\boldsymbol{D}} \boldsymbol{F}\left(\tau_{k}^{\prime}\right) \widetilde{\boldsymbol{E}}\left(\boldsymbol{K}_{s} \Delta_{s}-\overline{\boldsymbol{I}}\right) & -\boldsymbol{P}^{-1}
\end{array}\right]<0
$$

Inequation (A24) can be rewritten as:

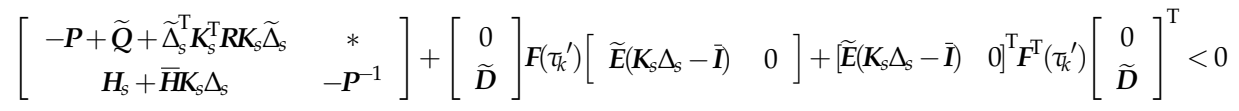

From Lemma 2, the following inequation is attained: 


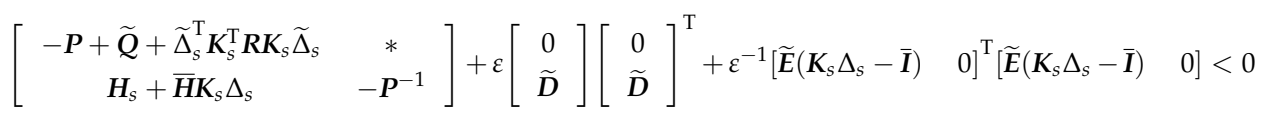

Using Lemma 1 again, the following inequation is obtained:

$$
\left[\begin{array}{cccc}
-\boldsymbol{P}^{-1}+\widetilde{\boldsymbol{Q}} & * & * & * \\
\boldsymbol{H}_{s}+\overline{\boldsymbol{H}} \boldsymbol{K}_{s} \Delta_{s} & -\boldsymbol{P}^{-1}+\varepsilon \widetilde{\boldsymbol{D}} \widetilde{\boldsymbol{D}}^{\mathrm{T}} & * & * \\
\widetilde{\boldsymbol{E}}\left(\boldsymbol{K}_{s} \Delta_{s}-\overline{\boldsymbol{I}}\right) & 0 & -\varepsilon \boldsymbol{I} & * \\
\boldsymbol{K}_{s} \Delta_{s} & 0 & 0 & -\boldsymbol{R}^{-1}
\end{array}\right]<0
$$

Note that inequation (A27) is not a feasible LMI. In order to obtain a feasible LMI, (A27) is pre-multiplied and post-multiplied by $\operatorname{diag}\left(\left[\begin{array}{llll}\boldsymbol{P}^{-1} & \boldsymbol{I} & \boldsymbol{I} & \boldsymbol{I}\end{array}\right]\right)$. Let $\boldsymbol{P}^{-1}=\boldsymbol{X}$, $\boldsymbol{Y}_{S}=K_{S} \Delta_{S} \boldsymbol{X}$, then we have:

$$
\left[\begin{array}{cccc}
-\boldsymbol{X}+\boldsymbol{X} \widetilde{\boldsymbol{Q}} \boldsymbol{X} & * & * & * \\
\boldsymbol{H}_{S} \boldsymbol{X}+\overline{\boldsymbol{H}} \boldsymbol{Y}_{s} & -\boldsymbol{X}+\varepsilon \widetilde{\boldsymbol{D}} \widetilde{\boldsymbol{D}}^{\mathrm{T}} & * & * \\
\widetilde{\boldsymbol{E}} \boldsymbol{Y}_{S}-\widetilde{\boldsymbol{E}} \boldsymbol{I} \boldsymbol{X} & 0 & -\varepsilon \boldsymbol{I} & * \\
\boldsymbol{Y}_{S} & 0 & 0 & -\boldsymbol{R}^{-1}
\end{array}\right]<0
$$

According to Lemma 1, inequation (20) holds. The proof is completed.

\section{References}

1. Zhang, X.-M.; Han, Q.-L.; Ge, X.; Ding, D.; Ding, L.; Yue, D.; Peng, C. Networked control systems: A survey of trends and techniques. IEEE/CAA J. Autom. Sin. 2019, 7, 1-17. [CrossRef]

2. Ge, X.; Yang, F.; Han, Q.-L. Distributed networked control systems: A brief overview. Inf. Sci. 2017, 380, 117-131. [CrossRef]

3. Zhang, D.; Shi, P.; Wang, Q.-G.; Yu, L. Analysis and synthesis of networked control systems: A survey of recent advances and challenges. ISA Trans. 2017, 66, 376-392. [CrossRef]

4. Papachristodoulou, A.; Jadbabaie, A.; Münz, U. Effects of Delay in Multi-Agent Consensus and Oscillator Synchronization. IEEE Trans. Autom. Control. 2010, 55, 1471-1477. [CrossRef]

5. De Persis, C.; Tesi, P. A Comparison Among Deterministic Packet-Dropouts Models in Networked Control Systems. IEEE Control. Syst. Lett. 2017, 2, 109-114. [CrossRef]

6. Qi, Y.W.; Liu, Y.H.; Niu, B. Event-Triggered H-infinity Filtering for Networked Switched Systems With Packet Disorders. IEEE Trans. Syst. Man Cybern. Syst. 2021, 51, 2847-2859. [CrossRef]

7. Liu, X.; Zhang, J.; Xia, Y. Co-Design of Quantization and Event-Driven Control for Networked Control Systems. IEEE Trans. Syst. Man Cybern. Syst. 2021, 51, 3103-3110. [CrossRef]

8. Nithya, V.; Sakthivel, R.; Alzahrani, F.; Ma, Y.K. Fault-tolerant H-infinity filtering for fuzzy networked control systems with quantisation effects. Int. J. Syst. Sci. 2020, 51, 1149-1161. [CrossRef]

9. Wu, C.; Zhao, X.; Xia, W.; Liu, J.; Başar, T. L2-gain analysis for dynamic event-triggered networked control systems with packet losses and quantization. Automatica 2021, 129, 109587. [CrossRef]

10. Cheng, L.-L.; Zhan, X.; Wu, J.; Han, T. An Optimal Tracking Performance of MIMO NCS with Quantization and Bandwidth Constraints. Asian J. Control. 2019, 21, 1377-1388. [CrossRef]

11. Fu, A.; McCann, J.A. Dynamic Decentralized Periodic Event-Triggered Control for Wireless Cyber-Physical Systems. IEEE Trans. Control. Syst. Technol. 2021, 29, 1783-1790. [CrossRef]

12. Zhu, S.; Liu, Y.; Lou, Y.; Cao, J. Stabilization of logical control networks: An event-triggered control approach. Sci. China Inf. Sci. 2020, 63, 1-11. [CrossRef]

13. Léchappé, V.; Moulay, E.; Plestan, F.; Han, Q.-L. Discrete predictor-based event-triggered control of networked control systems. Automatica 2019, 107, 281-288. [CrossRef]

14. Cervin, A.; Eker, J.; Bernhardsson, B.; Årzén, K.-E. Feedback-Feedforward Scheduling of Control Tasks. Real-Time Syst. 2002, 23, 25-53. [CrossRef]

15. Sha, L.; Rajkumar, R.; Lehoczky, J. Priority inheritance protocols: An approach to real-time synchronization. IEEE Trans. Comput. 1990, 39, 1175-1185. [CrossRef]

16. Baker, T.P. Stack-based scheduling of realtime processes. Real-Time Syst. 1991, 3, 67-99. [CrossRef]

17. Brockett, R. Stabilization of motor networks. In Proceedings of the 1995 34th IEEE Conference on Decision and Control, New Orleans, LA, USA, 13-15 December 1995. 
18. Rehbinder, H.; Sanfridson, M. Scheduling of a limited communication channel for optimal control. Automatica 2004, 40, 491-500. [CrossRef]

19. Wen, S.; Guo, G.; Li, Z. Binary sequence based dynamic scheduling and control co-design for cyber-physical systems. In Proceedings of the 2016 35th Chinese Control Conference (CCC), Chengdu, China, 27-29 July 2016; pp. 7216-7221.

20. Walsh, G.C.; Ye, H.; Bushnell, L.G. Stability analysis of networked control systems. IEEE Trans. Control Syst. Technol. 2002, 10, 438-446. [CrossRef]

21. Christmann, D.; Gotzhein, R.; Siegmund, S.; Wirth, F. Realization of Try-Once-Discard in Wireless Multihop Networks. IEEE Trans. Ind. Inf. 2013, 10, 17-26. [CrossRef]

22. Zhou, C.; Lu, H.; Ren, J.; Chen, Q. Co-design of dynamic scheduling and quantized control for networked control systems. J. Frankl. Inst. 2015, 352, 3988-4003. [CrossRef]

23. Mamduhi, M.H.; Hirche, S. Try-once-discard scheduling for stochastic networked control systems. Int. J. Control. 2019, 92, 2532-2546. [CrossRef]

24. Cao, Z.; Niu, Y.; Karimi, H.R. Sliding mode control of automotive electronic valve system under weighted try-once-discard protocol. Inf. Sci. 2020, 515, 324-340. [CrossRef]

25. Song, J.; Wang, Z.D.; Niu, Y.G.; Hu, J. Observer-based sliding mode control for state-saturated systems under weighted try-oncediscard protocol. Int. J. Robust Nonlinear Control 2020, 30, 7991-8006. [CrossRef]

26. Zhao, S.; Ji, Y. Model-dependent Scheduling and H-infinity Control Co-design for Networked Control Systems. Int. J. Control. Autom. Syst. 2021, 19, 969-979. [CrossRef]

27. Boyd, S.; Vandenberghe, L. Convex Optimization; Cambridge University Press: Cambridge, UK, 2004.

28. Kolmanovskii, V.; Myshkis, A. Applied Theory of Functional Differential Equations; Kluwer Academic Publisher: Boston, MA, USA, 1992 